\title{
Las relaciones de Venezuela con Ecuador y Bolivia en el ámbito de la cooperación internacional durante y después del gobierno de Hugo Chávez*
}

\author{
Mónica Vergara**
}

\section{Resumen}

La crisis en Venezuela ha dejado de lado el debate sobre su rol en la cooperación internacional. Sin embargo, resulta pertinente revisitar las relaciones del país caribeño con dos de los países andinos que durante y después del gobierno de Hugo Chávez jugaron el rol de aliados políticos. Este documento tiene como propósito describir el alcance de la cooperación de Venezuela con Bolivia y Ecuador, el contexto político y económico en el que se erige la cooperación y el posterior declive de los proyectos y acuerdos. Se destaca en este análisis el uso de petrodólares, el hiperpresidencialismo y una estrategia de soft balancing del presidente Hugo Chávez como sustento esencial para la cooperación, siendo estos mismos elementos los que marcaron su poca sostenibilidad. Los datos para la investigación se obtienen a través de una revisión y análisis crítico de documentos y declaraciones oficiales en torno a las relaciones de los tres países entre 2006 y 2018, así como de la literatura académica. Los resultados de esta investigación dejan ver el poco avance que tuvieron los proyectos (tanto bilaterales como en el marco de la Alianza Bolivariana para los Pueblos de Nuestra América - Tratado de Comercio de los Pueblos) incluso antes del recrudecimiento de la crisis económica de Venezuela.

Palabras claves: Venezuela - ALBA-TCP - Cooperación Sur-Sur - Ecuador - Bolivia

\section{Venezuela's relations with Ecuador and Bolivia in the field of international cooperation during and after Hugo Chávez administration}

\begin{abstract}
The crisis in Venezuela has left aside the debate about the role of this country in international cooperation field. However, there are still aspects to analyze, especially the relations with its Andean political allies during and after the government of Hugo Chávez. This paper aims to highlight important elements of cooperation between Venezuela, Bolivia and Ecuador, the political and economic context for cooperation and the subsequent decline of projects and agreements. It highlights how Venezuela used petrodollars, hyper-presidentialism and a soft balancing strategy as the main tools for foreign policy, which were elements get cooperation weaker. The data for the investigation were obtained through a critical review and analysis of documents and official declarations regarding the relations of three countries between 2006 and 2018, as well as the academic literature. The results of this research show the little progress the projects had (both bilateral and within the framework of the Bolivarian Alliance for the Peoples of Our America - Peoples' Trade Treaty) even before the resurgence of the economic crisis in Venezuela.
\end{abstract}

Keywords: Venezuela - ALBA-TCP - South-South Cooperation - Ecuador - Bolivia

TRABAJO RECIBIDO: 20/08/2019 TRABAJO ACEPTADO: 30/04/2020

\footnotetext{
* Este documento hace uso de una versión abreviada del segundo capítulo de la tesis de maestría del autor. El autor agradece los comentarios y observaciones a este trabajo por parte de los revisores anónimos.

${ }^{* *}$ Magíster en Relaciones Internacionales (Universidad Andina Simón Bolívar, Ecuador); Doctorando en Ciencias Sociales (Universidad de Salamanca, España). Docente-investigadora en la Pontificia Universidad Católica del Ecuador Sede Esmeraldas (PUCESE, Ecuador). Correo electrónico: lcdavergara@gmail.com
} 


\section{Introducción}

En la primera década del siglo XXI América Latina experimentó un importante giro hacia la izquierda, resurgiendo un espíritu tercermundista y logrando afianzar algunas propuestas de Cooperación Sur-Sur (en adelante CSS); especialmente como consecuencia del reacomodo geopolítico y geoeconómico que favoreció a los países del Sur global. Asimismo, organismos multilaterales comenzaron a prestar mayor atención a las experiencias de CSS, reconociendo el éxito de muchas prácticas y, lo más importante, reconociendo errores del sistema de la ayuda tradicional.

No obstante, el caso venezolano fue analizado con un enfoque dicotómico. La propuesta de cooperación del país caribeño en el siglo XXI fue vinculada, por un lado, a su condición rentista, de la cual se desprendía la financiación de proyectos con carácter geopolítico. El tinte radical del gobierno de Hugo Chávez fue considerado por Estados Unidos (y sus aliados) como un peligro para el statu quo de la región, teniendo en cuenta que los petrodólares le permitían al gobierno de Venezuela impulsar una propuesta de cooperación de gran alcance.

Asimismo, algunos analistas del tema posicionaron la cooperación venezolana bajo una mirada realista, interpretando la Alianza Bolivariana para los Pueblos de Nuestra América Tratado de Comercio de los Pueblos ${ }^{1}$ (en adelante ALBA-TCP) y los proyectos de cooperación bilateral como herramientas de soft balancing (Briceño, 2015; Corrales, 2009; Serbin y Serbin Pont, 2014; Toro, 2011), con miras a reducir la influencia hegemónica de Estados Unidos en Latinoamérica.

Por otra parte, hay análisis que en su momento valoraron la propuesta venezolana como un modelo a seguir (Aponte, 2014, 2015; Ayllón, 2012; Muhr, 2012, 2015; Roncal, 2013) ya que iba en consonancia con los principios de la CSS. Dichos principios establecen relaciones basadas en la horizontalidad y la complementariedad entre países y la inclinación hacia proyectos que se ajusten a las políticas de desarrollo de los países miembros (Ayllón, 2015) y no a los intereses económicos de los cooperantes.

Dentro de este paraguas de la cooperación horizontal, solidaria y sin condiciones (Organización de las Naciones Unidas, 2009; Comisión Económica para América Latina y el Caribe, 2010) se impulsó una agenda entre Venezuela y sus aliados políticos de la subregión andina, Ecuador y Bolivia, especialmente en el periodo de 2006-2012, cuando coinciden gobiernos progresistas y el boom de las commodities les permitía a sus economías extractivistas tener un mayor margen de autonomía en política exterior. Sin embargo, con el descenso de los precios del petróleo, la enfermedad de Hugo Chávez y su posterior muerte en 2013, la dinámica cooperativa e integracionista entre estos tres países comenzó a mostrar su cara más débil, lo que cambió el escenario de las relaciones y, sobre todo, de cooperación entre 2013 y 2018.

Este documento deriva de un seguimiento a las relaciones de Venezuela con Ecuador y Bolivia entre 2006 y 2018. La investigación tiene un alcance descriptivo y se lleva a cabo a través de una revisión documental contrastando la literatura y la información oficial de los tres países sobre CSS, ALBA-TCP y política exterior bajo el gobierno de Hugo Chávez y durante el periodo posterior a su muerte.

El objetivo esencial de este documento es describir el alcance de la cooperación de Venezuela con Bolivia y Ecuador, el contexto político y económico en el que se erige la CSS y el posterior declive de los proyectos. Por razones de espacio, sólo se han tomado para el análisis los proyectos de cooperación en los que se tuvo mayor acceso a la información. Si bien los programas sociales fueron los más relevantes en el ámbito discursivo, este documento, busca profundizar en otros aspectos que han sido menos explorados como el caso de los ejes energéticos o productivos.

\footnotetext{
${ }^{1}$ El ALBA-TCP es una propuesta de integración impulsada por Venezuela con los países de América Latina y el Caribe, a través de la cual se gestionó parte de la cooperación venezolana con un discurso apegado a los principios de la CSS: solidaridad, complementariedad y soberanía
} 
Vale aclarar que el documento no pretende ahondar en la descripción, el alcance o los debates sobre el ALBA-TCP como propuesta de integración. Sin embargo, este bloque se constituyó como eje principal para la cooperación venezolana, por lo cual, en los casos de Ecuador y Bolivia es relevante mencionar tanto las relaciones bilaterales como aquella cooperación que se intentó enmarcar dentro del ALBA-TCP.

La primera parte del documento aborda brevemente las características de la CSS de Venezuela durante el gobierno de Hugo Chávez, contrastando el discurso de horizontalidad, complementariedad y solidaridad que enfatizaba el país caribeño, con una perspectiva sustancialmente realista de la literatura sobre política exterior venezolana. El soft balancing y el uso de la renta petrolera son elementos centrales para este análisis. Asimismo, se aborda el hiperpresidencialismo como un aspecto crucial para el impulso de la agenda internacional venezolana, complementado con una personalidad carismática del fallecido presidente Hugo Chávez. Como un segundo apartado, se analizan los proyectos y acuerdos más relevantes de la cooperación de Venezuela con Ecuador y Bolivia, especialmente en el periodo 2006-2012 cuando coincidieron los gobiernos de Hugo Chávez, Evo Morales (Bolivia) y Rafael Correa (Ecuador).

Finalmente, se evalúan las relaciones de Venezuela con Ecuador y Bolivia entre 2013 y 2018, bajo un escenario de crisis económica y política para el país caribeño, sin Chávez y sin petrodólares. En este periodo se hace evidente el poco alcance e incluso la falta de materialización de varias de las propuestas.

\section{Aspectos relevantes de la CSS durante la era de Hugo Chávez}

La CSS supone el estrechamiento de lazos en las relaciones de los países en desarrollo y puede incluir cooperación relacionada con "intercambio de recursos, tecnología y conocimiento" (Mawdsley, 2019, p. 259). No se trata de un fenómeno nuevo, por el contrario, surge en el periodo de posguerra con la Conferencia de Bandung en 1955 y fue tomando fuerza con bloques posteriores (el Movimiento de los No Alineados, el G77) con los que nace la Cooperación Técnica entre Países en Desarrollo (CTPD). Sería la CTPD el primer antecedente de lo que hoy conocemos como CSS (López, 2014). Un momento clave para la CSS fue la demanda de un Nuevo Orden Económico Internacional (NOEI) que tendrá su principal reclamo en la Asamblea General Extraordinaria de las Naciones Unidas de 1974. Los principios de la CSS se consolidan en 1978 con la aprobación del Plan de Acción de Buenos (PABA).

No obstante, la CSS se ve mayormente fortalecida con la reconfiguración geoeconómica mundial del nuevo milenio y con la aparición de nuevas coaliciones, en las que países como China, Rusia, India, entre otros, apelan por un mundo multipolar, promoviendo cambios importantes en las relaciones de poder del sistema internacional. Por otro lado, el surgimiento de gobiernos progresistas en América Latina y la poca atención de Estados Unidos en la región, dio paso a nuevas propuestas de integración y cooperación, catalogadas con diversos adjetivos como contrahegemónicas (Muhr, 2011, 2012), posthegemónicas, postliberales (Riggirozzi, 2012; Sanahuja, 2009), revisionistas y antisistémicas (Briceño, 2013). Este punto de inflexión tuvo como referente en América Latina a la Revolución Bolivariana de Venezuela y los gobiernos de Brasil, Argentina, Bolivia, Ecuador, entre otros.

En el campo de la CSS y de la integración regional, Venezuela ha sido considerada un actor fundamental, característica que no es propia de la Revolución Bolivariana, sino un común denominador de los gobiernos venezolanos desde el establecimiento de la democracia (Domínguez, 2015). Este rol del país caribeño en el marco de la cooperación y la integración regional ha estado ligado a sus capacidades económicas en periodos de bonazas petroleras. Lo particular con el gobierno chavista fue su abierta determinación a contrarrestar la influencia estadounidense en la región a través de una propuesta de "integración alternativa" (ALBA$\mathrm{TCP}$ ), financiando ambiciosas iniciativas en un contexto político y económico favorable como lo fue el primer decenio del gobierno de Hugo Chávez. 
Propuestas como el ALBA-TCP y Petrocaribe posicionaron a Venezuela como uno de los más importantes cooperantes en la región en el siglo XXI. Los principios de solidaridad, horizontalidad y complementariedad, mismos de la CSS, son la consigna esencial de los acuerdos firmados por la República Bolivariana, tanto aquellos bilaterales como los enmarcados bajo el ALBA-TCP.

El discurso del gobierno chavista cautivó a grandes movimientos sociales a lo largo de América Latina e incluso fuera del continente, especialmente por el éxito de los programas sociales llevados a cabo en Venezuela con la colaboración del recurso humano cubano. Esto sirvió para legitimar la "internacionalización de las Misiones" a través del ALBA-TCP (Benzi, 2016).

No obstante, el posicionamiento de Venezuela como uno de los mayores cooperantes durante el primer decenio del milenio y "el récord de solidaridad" (Domínguez, 2015, p. 106) no fueron posibles sólo por los ingresos petroleros. Esto respondió, en gran medida, al modelo hiperpresidencialista que estableció el gobierno de Hugo Chávez. El expresidente consiguió apoyo casi irrestricto de las instituciones nacionales y tuvo acceso a los fondos provenientes de la renta petrolera de manera casi ilimitada para poder llevar a cabo su agenda de cooperación internacional. El hiperpresidencialismo ha sido un término comúnmente acuñado al modelo de gobierno del expresidente Chávez, sobre todo por el control que el ejecutivo tuvo sobre las instituciones, avalado por la Constitución Nacional (Penfold, 2010; Llanos y Nolte, 2016).

Andrés Serbin y Andrei Serbin Pont (2014, p. 292) señalan al respecto una marcada diferencia del gobierno de Chávez con respecto a sus antecesores en lo que concierne "a la formulación de una estrategia de proyección exterior sin mayores consultas con otros sectores y sin un activo involucramiento, así fuere técnico, de otros organismos institucionales, y sin la existencia de mecanismos de control o monitoreo por parte de la ciudadanía".

El hiperpresidencialismo desarrollado en Venezuela fue acompañado de un liderazgo carismático de parte del presidente Hugo Chávez. Diversos análisis han profundizado en este tema, basados en los supuestos de la teoría weberiana, dónde se contempla al líder carismático como un personaje lleno de atributos extraordinarios, que lo conectan de manera especial con sus seguidores, generando de esta forma procesos transformadores (Arenas, 2016; Villarroel y Ledezma, 2007). Los análisis han coincido en que el carisma del presidente Hugo Chávez fue un aspecto determinante para el posicionamiento de su gobierno en el ámbito doméstico e incluso a nivel internacional.

En relación a las cifras reales sobre los desembolsos de cooperación internacional por parte de la Revolución Bolivariana no han sido explícitas, especialmente por el manejo discrecional de la información que hubo en muchos de los casos. Después de la muerte de Hugo Chávez ha sido incluso más difícil medir la dinámica de Venezuela en el ámbito de la cooperación internacional, sin embargo, un informe del Consejo Económico y Social de las Naciones Unidas (citado por The Reality of Aid, 2010, p. 36), ya posicionaba en aquel entonces a Venezuela en el tercer lugar -después de Arabia Saudita y China- dentro de los principales cooperantes (no pertenecientes al Comité de Ayuda al Desarrollo), superando el 0,7\% de la Renta Nacional Bruta; meta que deberían cumplir los países miembros de la Organización para la Cooperación y el Desarrollo Económico (OCDE).

Asimismo, en un informe de la Secretaría General Iberoamericana (2008, p. 6) se menciona un desembolso aproximado de 15 mil millones de dólares por parte del gobierno chavista para ayuda humanitaria y otros 15 mil millones de dólares de desembolso bajo los programas de Petrocaribe.

Por otro lado, un informe de un think tank estadounidense denominado The Atlantic Council, afirma que entre 2001 y 2013 Petróleos de Venezuela (PDVSA) desembolsó alrededor de 3 mil millones de dólares anuales para cooperación internacional (Goldwyn \& Gill, 2014, p. 7). En otras investigaciones académicas se menciona que entre 1999 y 2009, Venezuela aportó unos 36,4 mil millones aproximadamente entre créditos concesionales que incluyen acuerdos de cooperación petrolera y financiamiento (Domínguez, 2015; Romero y Curiel, 2009). 
Esta falta de transparencia trae a colación la otra cara de la moneda. Más allá de si se trató o no de un genuino y exitoso caso de CSS, el accionar de Venezuela se ha estudiado dentro de un marco realista de las Relaciones Internacionales, destacando una política exterior basada en el soft balancing. En la mayoría de los casos, los analistas que se inclinan por esta explicación hacen referencia al ALBA-TCP, ya que el gobierno de Hugo Chávez proyectó a este bloque como el andamio para los proyectos de cooperación internacional. La literatura sobre política exterior venezolana ha profundizado en el concepto de soft balancing promovido por Robert Pape (2005), el cual se refiere al uso de herramientas no militares para contrarrestar el poder hegemónico.

De acuerdo con la teoría desarrollada por Pape (2005) la política exterior basada en el soft balancing no implica desafiar necesariamente al poder militar del líder unipolar, pero sí logran contrarrestar la aplicación de su poderío. En este sentido, Pape explica que el uso de elementos de cooperación no militares contribuye a que los países secundarios (dentro de un sistema internacional unipolar) puedan construir una convergencia de intereses que les beneficien (2005, p. 18). En el caso venezolano y, concretamente, en el gobierno de Hugo Chávez, la cooperación (basada en una cantidad ingente de petrodólares) fue usada como una estrategia "suave" para desafiar el poder hegemónico de Estados Unidos.

Al respecto, Javier Corrales (2009) hace referencia al caso venezolano enmarcado en "el uso de generosos donativos en el extranjero, salpicado de un discurso favorable a los pobres y propensos a la distribución" (p. 97). Esta estrategia enmarcada en la petrodiplomacia venezolana fue efectiva gracias a los ingresos petroleros provenientes de una bonanza sin precedente y de la triangulación con Cuba.

José Briceño Ruiz (2015) acompaña la visión de Corrales sobre la estrategia venezolana de promover "una diplomacia social y una diplomacia petrolera" con fines estratégicos para "balancear la influencia de Estados Unidos en la América Latina y el Caribe" (p. 188). Sin duda, los programas desarrollados en áreas sociales sirvieron en su momento "para establecer equilibrios frente al poder hegemónico" (p. 184).

Alfredo Toro (2011) agrega de manera más explícita que, aunque Venezuela y, concretamente, los países miembros del ALBA no están en capacidad de generar una alteración real dentro del orden internacional, el empleo de ciertas estrategias de "poder suave" buscaban "retardar, frustrar y debilitar los ámbitos de dominio de los Estados Unidos" (p. 160). Una manera muy clara de entender las intenciones de Venezuela de contrarrestar el poder hegemónico está precisamente en las propuestas de cooperación en el eje energético, un espacio estratégico para el país caribeño.

Desde una perspectiva quizá menos debatida, el caso venezolano muestra que en la CSS también es posible encontrar algunas de las problemáticas de la Cooperación Norte-Sur (CNS), es decir, la ayuda ligada a intereses nacionales, sean estos económicos, comerciales o políticoideológicos. En esta misma línea de ideas, algunos académicos han explicado cómo el gobierno chavista ha usado su maquinaria rentista para asegurarse apoyo en espacios de concertación política (Caballero Santos, 2012; Cobo, 2008; Corrales \& Penfold, 2011).

Lo relevante del caso, es que Venezuela se convirtió en una amenaza al status quo, en principio por una posición contestataria del gobierno de Hugo Chávez contra la hegemonía estadounidense, pero además por sus capacidades económicas en un momento histórico para el país.

Sin embargo, el rentismo petrolero de Venezuela marcó desde el principio una limitante para la propuesta regional del gobierno chavista. Ante un escenario tan inestable como el que representan las commodities resulta difícil sostener un plan de cooperación como el propuesto por Venezuela en sus años dorados. Ciertamente, la muerte de Hugo Chávez contribuyó a la precipitación del fracaso de la CSS venezolana en el siglo XXI. Esta realidad se puede evidenciar, en casos menos estudiados, como Ecuador y Bolivia. 


\section{Relaciones de cooperación Venezuela-Ecuador-Bolivia periodo 2006-2012}

Las relaciones internacionales de Venezuela y sus intereses geopolíticos bajo los gobiernos precedentes al de Hugo Chávez no se dirigían a la región andina, de hecho, la cooperación que impulsaba Venezuela apuntaba mayormente hacia la región caribeña y hacia algunos países centroamericanos ${ }^{2}$.

Con el nuevo milenio y ante un escenario global convulsionado se abrían paso nuevas alianzas que se vieron reflejadas con la llegada de Evo Morales a la presidencia de Bolivia en 2006 y más tarde Rafael Correa en Ecuador en 2007. Chávez, por su parte, construía un discurso integracionista más ambicioso, la consolidación de la "Patria Grande".

Los recursos naturales les permitieron a los gobiernos progresistas de Ecuador y Bolivia tener un gran margen de acción en sus políticas públicas (Vergara, 2016, p. 52), logrando reducir en gran medida los niveles de pobreza y analfabetismo (Aponte, 2014; Ponce y Onofa, 2009; Roncal, 2013; UNESCO, 2013). No obstante, los cambios en Ecuador y Bolivia fueron aún más evidentes. De acuerdo con el Instituto Nacional de Estadística de Bolivia (2018) entre 2006 y 2017, la pobreza bajó de 59,6\% a 36,4\%. Ribando (2008) también explica que la nacionalización de los hidrocarburos en Bolivia consiguió un incremento sin precedentes de unos 1.57 billones de dólares en 2007 en comparación con 173 millones de dólares que obtuvo en 2002, gracias a estos ingresos y una mayor redistribución se logran grandes avances en materia social.

En el caso de Ecuador, aunque con matices políticos y realidades distintas, también se percibió un gran avance en materia social. De acuerdo con un informe del Instituto Nacional de Estadísticas de Ecuador (2017) "en el periodo comprendido entre diciembre 2007 y diciembre 2016 la tasa de pobreza se reduce 13,8 puntos porcentuales, de $36,7 \%$ a $22,9 \%$ ".

Bajo un escenario económicamente favorable, se establecieron las bases políticas de los gobiernos progresistas de Ecuador, Bolivia y Venezuela para llevar adelante una propuesta de integración en la región, basada en el éxito que tuvo la alianza Cuba-Venezuela en materia de cooperación donde según Cusack (2015) prevaleció la complementariedad. Los acuerdos, actas de intención y declaraciones firmadas por Ecuador y Bolivia con la República Bolivariana de Venezuela hacen hincapié en el ser humano, explicando que se les debe considerar el "factor fundamental de la economía" (Acosta, 2010, p. 23).

Las propuestas que se impulsaban desde Latinoamérica y especialmente desde Venezuela, Ecuador y Bolivia, iban dirigidas a la búsqueda de "agendas comunes" y de una "integración alternativa" (Aponte, 2014, 2015; Roncal, 2013) con énfasis en la autodeterminación y la solidaridad de los pueblos. En esencia la pretensión parecía sostenerse en la construcción de "algo propio", sin injerencias de potencias hegemónicas y sobre todo "afín a los intereses de los países de Latinoamérica" (Falconí, 2010).

Todas estas características (ambiciosas y utópicas dependiendo desde donde se le mire) despertaron interesantes debates académicos y políticos sobre el rol de Venezuela en la CSS y, de manera muy general, se vincularon con las realidades de Ecuador y Bolivia en un nuevo marco de integración más bolivariano.

Bajo el paraguas de la solidaridad, se firmaron convenios, acuerdos, actas de intención que abarcan proyectos en áreas estratégicas: productividad, comercio y energía; respondiendo esta última al modelo extractivista de las tres naciones. Desde el gobierno de Venezuela se trató de promover la mayoría de los convenios dentro del marco del ALBA-TCP; sin embargo, las evidencias muestran que hubo mayor inclinación por los acuerdos bilaterales.

\footnotetext{
${ }^{2}$ Casos como el Acuerdo de Puerto Ordaz (1975) y el Acuerdo de San José (1980) dan muestras de los campos de acción en los que cooperaba la Venezuela de la IV República.
} 


\subsection{Cooperación Venezuela-Bolivia}

Bolivia se ha caracterizado por ser un país con alta dependencia de la cooperación internacional NS y ha sido visto como el "ratón de laboratorio" (De Grave, 2007) de los donantes tradicionales. No obstante, desde el gobierno de Morales esta situación fue revertida de manera significativa. De acuerdo con datos oficiales "en el periodo 1999-2005 la inversión pública financiada con recursos externos alcanzó un promedio de $55 \%$, en tanto que en el periodo 2006-2012, esta proporción se ha reducido a 31\%" (Viceministerio de Inversión Pública y Financiamiento Externo, 2013). Asimismo, la Organización para la Cooperación y el Desarrollo Económico (citado por Paz, 2016, p. 1) establece que en el periodo 2006-2013 la participación de Bolivia en la AOD destinada a países en desarrollo se redujo de 0,26 a 0,13\%.

Por otra parte, Bolivia fue uno de los países con los que el gobierno de Venezuela mantuvo relaciones políticas más cercanas en Sudamérica. Venezuela sirvió como apoyo por un tiempo para evadir la dependencia hacia las ONG y las agencias internacionales de cooperación (Vergara, 2016).

Dentro del eje social (uno de los más importantes) se firmaron diversos acuerdos bilaterales entre Venezuela y Bolivia que abarcaban áreas deportivas, salud, educación universitaria, entre otros. Por supuesto, "la internacionalización de la Misiones" (Benzi, 2016) tuvo un impacto importante en Bolivia, contando con la participación de Cuba. La investigación en documentos oficiales de Bolivia arroja que gran parte de la cooperación de Venezuela con este país consistió en préstamos y donaciones destinadas a desarrollo social, varios de ellos dentro del marco del ALBA-TCP (Vergara, 2016, p. 77).

De acuerdo con el informe del ALBA-TCP (2014) entre 2006 y 2014 alrededor de 539.276 personas fueron atendidas por la Misión Milagro. En la Misión Moto Méndez (para personas con discapacidad) se atendieron unas 82.087 personas. En Bolivia también se alfabetizaron unas 826.865 personas. Además, 551 personas egresaron de medicina integral después de haber sido becados dentro de los convenios del ALBA-TCP.

Los acuerdos que se firmaron entre Venezuela y Bolivia de manera bilateral siguieron el mismo sistema que se había planteado dentro del ALBA-TCP, la división de ejes estratégicos en los que se destacaba la soberanía de los países. Es así como se establecen los cuatro ejes más importantes: eje social y del conocimiento, desarrollado a partir del Convenio Básico de Cooperación Técnica entre el Gobierno de la República Bolivariana de Venezuela y el Gobierno de la República de Bolivia firmado en 2006; eje de soberanía energética, que tuvo como base la suscripción del Acuerdo sobre Cooperación en el Sector Energético el 23 de enero de 2006; eje de soberanía comercial, teniendo como punto de partida el Acuerdo Comercial de la Alternativa Bolivariana de los Pueblos de Nuestra América y Tratado Comercial de los Pueblos (ALBATCP) suscrito por Venezuela, Cuba y Bolivia en 2006 y que fue extendido en 2011 luego de la salida definitiva de Venezuela de la Comunidad Andina. Asimismo, el eje de seguridad y defensa que nace con el Acuerdo Complementario al Convenio Básico de Cooperación Técnica entre la República de Bolivia y la República Bolivariana de Venezuela en Materia de Defensa firmado en 2006. A continuación, se señalan algunos de los proyectos y de las características más relevantes de dichos acuerdos.

El programa "Bolivia Cambia, Evo Cumple" ${ }^{3}$ fue el más polémico y, al mismo tiempo, más importante de los esquemas financiados por el gobierno chavista. Bajo este espacio se movieron gran parte de los fondos otorgados por Venezuela, sin llegarse a conocer a ciencia cierta cuál fue el monto real del desembolso. En 2011, un medio de comunicación boliviano explicaba que en el periodo 2006-2010 "Bolivia Cambia, Evo cumple" ejecutó más de 4.000 obras $^{4}$. Por otra parte, Evo Morales reconoció en 2008 que los recursos recibidos como parte de la cooperación venezolana eran tramitados directamente por la embajada del país caribeño a fin

\footnotetext{
${ }^{3}$ Este programa fue lanzado por el gobierno de Evo Morales en 2006 como plan de asistencia a los municipios y a los movimientos sociales de Bolivia

${ }^{4}$ Venezuela ya no financia obras del programa "Evo cumple", La Patria, 19 de julio de 2011, La Paz. Recuperado de http://www.lapatriaenlinea.com/?nota=75822. Consulta: 31 de marzo de 2015
} 
de evitar trámites burocráticos del Estado boliviano ${ }^{5}$. De alguna manera estas declaraciones servían para entender el nivel de discrecionalidad con el que el gobierno venezolano manejaba sus proyectos con Bolivia (Vergara, 2016, p. 77).

En cuanto a la reconfiguración energética, YPFB Petroandina $\mathrm{SAM}^{6}$ se destaca como una de las más relevantes propuestas. La misma nace en 2007 como una empresa mixta entre Venezuela y Bolivia, constituida con el $60 \%$ de capital boliviano (Yacimientos Petrolíferos Fiscales Bolivianos-YPFB) y 40\% de capital venezolano (PDVSA-Bolivia) (Petroandina, 2014). El presidente de Petroandina, Jaime Arancibia, afirmaba en 2013 en un medio de comunicación que el proyecto era "geoestratégico y geopolítico, gracias a la visión de Morales y Chávez". Estimando además que con la explotación del bloque Lliquimuni se "triplicarían las reservas petroleras de Bolivia" (citado por Paredes, 2013).

En cuanto a los asuntos comerciales, si bien no es un ámbito propiamente de la CSS, Venezuela lo incluyó como un eje estratégico dentro del ALBA-TCP, enmarcándolo también dentro de la cooperación con sus pares andinos. La cooperación venezolana consistió en proveer a los pequeños y medianos productores bolivianos de ayudas relevantes dentro del marco del ALBA-TCP. El sector textilero fue uno de los mayores beneficiarios después de firmar un acuerdo de cooperación en 2009. Se estableció que lo pequeños productores de textiles iban a tener la garantía en el mercado venezolano de una regla de origen de 70/30, es decir, 70\% de componente importado se aceptaría en los productos textiles exportados a Venezuela y $30 \%$ producto nacional (Guzmán, 2011). Bajo este acuerdo se creó en Bolivia un fidecomiso que ascendía a 10.000.000 de dólares para otorgar liquidez a los empresarios que exportaban sus productos al mercado venezolano. Este fondo era con financiamiento del Banco del ALBA (Decreto 196, 2009).

Por otra parte, Pablo Guzmán, quien fuera el viceministro de Comercio e Integración de Bolivia en 2011, declaraba en ese mismo año que "entre 2008 y 2010 el sector textilero alcanzó un monto por exportaciones de 55 millones 800 mil dólares, logrando Bolivia duplicar en el mercado venezolano, lo que perdió con la ATPDEA ${ }^{7 \text { " }}\left(\right.$ Guzmán, 2011) ${ }^{8}$. Estas declaraciones aparentemente fueron sobredimensionadas y los mismos gremios de textiles las desmintieron cuando salieron a flote limitaciones de Venezuela para convertirse en un potencial mercado para el ámbito textilero (Vergara, 2016, p. 85).

Hubo otros tantos proyectos de carácter bilateral que estaban contemplados dentro de la cooperación venezolana con Bolivia, como por ejemplo la construcción conjunta de una fábrica de Litio con propósitos industriales. Según declaraciones del viceministro9, Venezuela presentaba la propuesta más seria y justa de todas las recibidas hasta entonces. Sin embargo, este proyecto (al igual que la mayoría de los proyectos de inversión venezolana) fue cancelado o retomado con fondos de otros socios. Asimismo, se firmaron actas de intención para llevar a cabo proyectos de soberanía alimentaria (incluyendo la construcción de una Gran-Nacional)

\footnotetext{
${ }^{5} \mathrm{Si}$ es ratificado en referendo garantizará las autonomías, promete Evo Morales, Rebelión, 14 de julio de 2008. Recuperado de https://rebelion.org/si-es-ratificado-en-referendo-garantizara-las-autonomiaspromete-evo-morales/. Consulta: 31 de marzo de 2015

${ }^{6}$ Petroandina era una de las empresas propuestas dentro del marco de Petroamérica. Este último era un megaproyecto del gobierno de Venezuela, que consistía básicamente en una Gran-Nacional constituida por las empresas estatales de la región.

7 Andean Trade Promotion and Drug Eradication Act (Ley de Preferencias Arancelarias Andinas y Erradicación de la Droga) ATPDEA, por sus siglas en inglés, consistió en un sistema de preferencias comerciales implementado en la década de los ' 90 por Estados Unidos para que exportaciones de algunos países andinos entraran libres de aranceles a sus mercados a cambio de cooperar en contra de la producción y tráfico ilícito de drogas.

${ }^{8}$ Bolivia y Venezuela amplían el acuerdo comercial TCP por otros 5 años, Los Tiempos, 01 de abril de 2011, La Paz. Recuperado de https://www.lostiempos.com/actualidad/economia/20110401/boliviavenezuela-amplian-acuerdo-comercial-tcp-otros-5-anos. Consulta: 31 de marzo de 2015

9 Viceministro boliviano destaca propuesta venezolana para fabricar baterías de Litio, Correo del Orinoco, 02 de abril de 2011, Caracas. Recuperado de http://www.correodelorinoco.gob.ve/viceministroboliviano-destaca-propuesta-venezolana-para-fabricar-baterias-litio/. Consulta: 30 de marzo de 2015.
} 
entre Venezuela y Bolivia dentro del marco del ALBA-TCP (Ley 167, 2011), la cual no se materializó.

La seguridad y defensa constituyó otro de los ejes de cooperación de Venezuela con Bolivia $^{10}$. En estos convenios sólo se mencionan aspectos de cooperación técnica; sin embargo, fuentes alternas afirman que hubo también financiamiento venezolano para la adquisición de armamento y aviones en Bolivia ${ }^{11}$ (Asociación Civil Control Ciudadano, 2016, p. 15-16). La polémica no se hizo esperar, especialmente de parte del ala opositora al gobierno de Morales, quienes hacían hincapié en las injerencias que mantenía el gobierno de Chávez en asuntos internos de Bolivia (Vergara, 2016, p. 89).

Finalmente, las "Casas del ALBA" forman parte de un aspecto que poco se toca en los análisis sobre el ALBA-TCP. De acuerdo con Petersen (2012) funcionaban en el ámbito cultural y también en lo social, apoyando al proceso de cambio del gobierno de Evo Morales y los programas sociales del ALBA-TCP. Lo más reciente recopilado en la investigación muestra la inclusión de los estudiantes de secundaria en Bolivia y que se hace llamar Cátedra Libre Comandante Hugo Chávez ${ }^{12}$.

Las "Casas ALBA" supusieron un trabajo con las organizaciones de base, los movimientos sociales de Bolivia, apoyados plenamente por el gobierno de la República Bolivariana de Venezuela. No existe mucha información oficial ni académica sobre las actividades que se llevan a cabo dentro de las "Casas ALBA", tampoco del financiamiento (Vergara, 2016, p. 90).

Hasta 2018 -y lejos de evidenciarse éxito en los proyectos conjuntos de Venezuela y Bolivia, sobre todo en materia energética, económica o comercial- el discurso de Evo Morales con respecto a la República Bolivariana seguía siendo abiertamente afín y leal al proyecto chavista. En los espacios de concertación política y en organismos internacionales Bolivia mantuvo su apoyo al gobierno de Maduro, lo que de alguna manera se relaciona con los efectos de la cooperación política venezolana.

\subsection{Cooperación Venezuela-Ecuador}

Al igual que con Bolivia, el gobierno de Venezuela aprovechó su auge de petrodólares para firmar entre 2007 y 2011 un total de 114 acuerdos con Ecuador que se enmarcaron en seis ejes estratégicos: eje de soberanía social; eje de soberanía del conocimiento; eje de soberanía energética; eje de soberanía productiva; eje de soberanía financiera y comercial; eje de soberanía de seguridad y defensa ${ }^{13}$. Los acuerdos en el ámbito social se desarrollaron en cooperación triangular con Cuba y en mayor medida dentro del marco ALBA-TCP (Vergara, 2016, p. 60).

\footnotetext{
${ }^{10}$ Bolivia y Venezuela acentúan cooperación bilateral en el área de Defensa, Infodefensa, 5 de junio de 2013, La Paz. Recuperado de https://www.infodefensa.com/latam/2013/06/05/noticia-bolivia-yvenezuela-acentuan-cooperacion-bilateral-en-el-area-de-defensa.html. Consulta: 12 de noviembre de 2019

${ }^{11}$ De acuerdo con datos de una investigación en noviembre de 2007, el presidente Evo Morales entregó un cheque por 6.316.448 dólares al comandante en jefe de las Fuerzas Armadas, proveniente de fondos venezolanos. La fuente indica que Morales reconoció públicamente que dichos fondos serían usados para mejoras en infraestructura cuartelaria de 125 unidades militares. Asimismo, se afirmaba en aquel entonces que Venezuela aportaría diez millones de dólares para ampliar la Escuela Naval de Bolivia y otros 500 mil dólares para la construcción de un buque multipropósito destinado a la Armada de Bolivia y puesto en servicio en febrero de 2009 (Asociación Civil Control Ciudadano, 2016, p. 3).

12 Abordará pensamiento del líder de la Revolución Bolivariana. Inicia Cátedra "Hugo Chávez" en Bolivia, Correo del Orinoco, 27 de mayo de 2015, Cochabamba. Recuperado de http://www.correodelorinoco.gob.ve/inicia-catedra-\%E2\%80\%9Chugo-chavez\%E2\%80\%9D-bolivia/.

Consulta: 15 de marzo de 2016

${ }^{13}$ Información extraída de http://www.ecuador.embajada.gob.ve/. Consulta: 18 de diciembre de 2014
} 
La cooperación de Venezuela con Ecuador en el eje social, al igual que en Bolivia, se dio a través de una réplica de las Misiones, en alianza con Cuba, siendo estos los proyectos más exitosos en torno a la solidaridad que promueve la CSS y los únicos que se fijaron explícitamente dentro del marco del ALBA-TCP; los demás, como por ejemplo, acuerdos en educación, desarrollo científico, acuerdos en materia deportiva, entre otros, funcionaron como acuerdos bilaterales, aunque bajo el paraguas discursivo de la cooperación ALBA-TCP.

Ecuador fue declarado en 2009, por la UNESCO, como territorio libre de analfabetismo, al igual que Venezuela, Nicaragua y Bolivia. Aunque en declaraciones del entonces ministro de Educación, Raúl Vallejo (2009) se aclaró el término, explicando que se trataba de "Ecuador Patria Alfabetizada" y no libre de analfabetismo. El informe del ALBA-TCP (2014) indica que en Ecuador se alfabetizaron 372.185 personas bajo los programas sociales de este bloque.

Uno de los programas que tuvo mayor impacto en Ecuador fue la Misión Milagro, donde se daba atención a las personas con problemas de la vista y donde fueron atendidas unas 144.134 personas. Otro programa importante fue la Misión Manuela Espejo, donde se atendieron y censaron 294.803 personas con discapacidad. Todos estos datos hacen referencia a los programas financiados por Venezuela, con la ayuda de Cuba y que se impulsaron dentro del marco del ALBA-TCP (ALBA-TCP, 2014).

En cuanto al eje de soberanía energética se firmaron acuerdos para llevar a cabo ambiciosos proyectos, entre ellos, la construcción de la Refinería del Pacífico Eloy Alfaro RDP Compañía de Economía Mixta. Según datos oficiales, esta refinería tendría una capacidad de refinamiento de 300 mil barriles diarios para la producción de gasolina, diésel, jet fuel, corrientes petroquímicas (benceno y mezcla de xilenos), polipropileno, azufre y coque $\mathrm{e}^{14}$.

Este proyecto tenía un significado geoestratégico para Venezuela, por su necesidad de salir al Pacífico, además del innegable beneficio que representa también para Ecuador tener la refinería (Vergara 2016, p. 67). No obstante, la alianza con Venezuela para la construcción de la refinería no resultó sostenible, lo que motivó la inclusión de nuevos socios. A través documentos oficiales ${ }^{15}$ se pudo constatar que China National Petroleum Corp. (CNPC) y el banco chino Industrial and Commercial Bank of China (ICBC) fungían como accionistas del proyecto desde 2013, dejando a Petróleos de Venezuela (PDVSA) un mínimo de participación (Contraloría General del Estado de la República de Ecuador, 2014).

El intercambio de crudos por derivados y lubricantes propuesto por el gobierno de Venezuela a Ecuador desde 2006 constituye otro acuerdo relevante, ya que tuvo resultados positivos en sus primeros años, según fuentes oficiales. Durante el V Encuentro de presidentes en 2009 las autoridades afirmaban que Ecuador había alcanzado un margen de ahorro de 230 millones de dólares entre 2007 y 2009 (Ministerio de Relaciones exteriores y Movilidad Humana de la República del Ecuador, 2009).

Por otra parte, existen otras investigaciones que revelan irregularidades en este acuerdo, entre ellas un beneficio triangular para PDVSA. De acuerdo con una investigación de Villavicencio (2013), este convenio generó en realidad beneficios triangulares para el gobierno de Venezuela y sus socios privados.

Otro de los proyectos de gran envergadura en el eje energético fue la construcción de la Gran-Nacional Minera Mariscal Sucre CEM, la cual nace en 2010 producto de "un reenfoque comercial con la intención de fortalecer las relaciones de los países latinoamericanos y caribeños, a través del desarrollo sustentable, niveles de desarrollo económico y mejoras de cooperación" (Gran-Nacional Minera, 2014, p. 3). Este proyecto se firma de manera bilateral, aunque dentro de la propuesta de modelo Gran-Nacional del ALBA-TCP.

Esta última empresa es, quizá, una de las pocas que se logró materializar. Su actividad se basó en proyectos de minería a cielo abierto que estuvieron forjándose durante alrededor de

\footnotetext{
${ }^{14}$ Información extraída de http://www.rdp.ec/. Consulta: 12 de febrero de 2015

15 En el mismo documento de la Contraloría General del Estado consta que al 31 de diciembre de 2012, PDVSA Ecuador presentaba un déficit de 104 millones de dólares en el desembolso de sus obligaciones.
} 
seis años. De acuerdo con datos proporcionados por una vocera de la misma empresa, el objetivo era contribuir al cambio de la matriz productiva de Ecuador (citado por Vergara, 2016, p. 65).

En este sentido, se llevaron a cabo dos proyectos (ambos en la zona Norte de Ecuador); uno de ellos en Mompiche que buscaba, mediante la obtención de hierro y titanio, contribuir a la industria siderúrgica del país. Mientras que con el proyecto "El Reventador" se buscaba favorecer la industria agrícola gracias a los fosfatos (Gran-Nacional Minera, 2014, p. 3).

En términos productivos y comerciales, uno de los asuntos de mayor importancia, de acuerdo con fuentes oficiales, era cooperar mutuamente para conseguir la seguridad alimentaria y mecanismos de comercialización favorables para los países considerando la complementariedad como piedra angular. Bajo este paraguas, propio también de la CSS, se firmaron acuerdos bilaterales para cooperación técnica e intercambio de productos, además de las ambiciosas propuestas de Gran-Nacionales de Cacao, de Pesca y Acuicultura. Sin embargo, dichas propuestas quedaron en "Actas de entendimiento" según arroja la recopilación de información oficial desde la página web del Ministerio de Relaciones Exteriores de la República del Ecuador ${ }^{16}$.

En 2011, durante el IX Encuentro Binacional de presidentes, se firmaron unas 12 actas de proyectos de cooperación bilateral, que no llegaron a concretarse, con excepción de la creación de un fidecomiso o Fondo Ecuador-Venezuela para el Desarrollo (FEVDES) ${ }^{17}$ entre ambos países, al que Venezuela aportaría 50 millones de dólares y Ecuador 16 millones de dólares, cuyo memorándum de entendimiento ya se había firmado el 7 de octubre de 2009 (Ministerio de Relaciones Exteriores y Movilidad Humana de la República del Ecuador, 2009).

Cabe destacar, además, que éste fue el último encuentro entre los presidentes de Ecuador y Venezuela. Debido a la enfermedad del presidente venezolano la dinámica de los encuentros binacionales de presidentes, que se llevaban a cabo de manera continua, cesó. El presidente Maduro se comprometió en 2013 a retomar la agenda ${ }^{18}$, pero no existen evidencias de algún otro encuentro de este tipo (Vergara, 2016, p. 73).

Con respecto al ámbito comercial, también se firmaron algunos acuerdos bilaterales entre Venezuela y Ecuador que en algún momento se matizaron como un eje de cooperación. Al respecto, es importante mencionar que la balanza comercial fue distorsionada con una serie de transacciones fraudulentas que se realizaron entre Venezuela y Ecuador a través del uso del Sistema Unitario de Compensación Regional (SUCRE), propuesta que también estaba enmarcada en la cooperación financiera ALBA-TCP y en la Nueva Arquitectura Financiera Regional que impulsaban los gobiernos progresistas de la región y que nos lleva también al eje financiero.

Esta proposición dio indicios de una experiencia exitosa entre Venezuela y Ecuador, precisamente por el auge que tuvo el uso del SUCRE entre 2011 y 2013 en los intercambios comerciales de estos dos países (Secretaría Ejecutiva del Consejo Monetario Regional del SUCRE, 2013). Sin embargo, en 2013 el Fiscal General del Estado ecuatoriano, Galo Chiriboga, dio a conocer que gran parte de dichos intercambios se habían llevado a cabo entre "empresas de maletín" (Citado por Benzi, Guayasamín y Vergara, 2016)

A diferencia de Bolivia, las donaciones no fueron muy comunes en las relaciones de Venezuela con Ecuador durante la alianza revolucionaria, excepto en el eje militar (Asociación

\footnotetext{
${ }^{16}$ Información extraída de http://sitrac.cancilleria.gob.ec. Consulta: 26 de abril de 2016

${ }^{17}$ Cabe destacar que este mismo fondo fue usado para financiar la Gran-Nacional Minera.

${ }^{18}$ Rafael Correa en Venezuela, rueda de prensa junto al Presidente Nicolás Maduro, TeleSUR, 29 de julio de 2013. Recuperado de https://www.youtube.com/watch?v=TwuWtwd-ID0. Consulta: 13 de agosto de 2016

${ }^{19}$ Se les conoce como empresas de maletín aquellas actividades ilícitas, bajo las cuales se llevan a cabo procesos para registrar empresas ficticias. El fin último de una "empresa de maletín" es obtener divisas y sacarlas luego del país.
} 
Civil Control Ciudadano, 2016, p. 23; Ministerio de Relaciones Exteriores y Movilidad Humana de la República del Ecuador, 2009) ${ }^{20}$. Este eje parte del Acuerdo de Cooperación TécnicoMilitar firmado en octubre de 2009. Dicho acuerdo y el donativo previo despertaron cierta suspicacia ya que se llevaba a cabo justamente después que Ecuador hubiese enfrentado una violación a su soberanía territorial por parte de Colombia bajo el Plan Colombia (cooperación estadounidense) (Vergara, 2016, p. 76).

\section{La era post-Chávez, periodo 2013-2018}

En 2009 la debacle de los precios del petróleo afectaba ya la economía venezolana y desde 2011 la enfermedad de Hugo Chávez restó dinamismo a la agenda de cooperación de Venezuela, al menos con Ecuador y Bolivia. El mismo Rafael Correa reconoció en 2013, durante una visita oficial a Venezuela, que los proyectos habían perdido fuerza por la falta de seguimiento de los presidentes de ambas naciones. Esto indica que el éxito dependía de dos factores: el hiperpresidencialismo y el carisma de Hugo Chávez y los petrodólares venezolanos (Vergara, 2016).

Evo Morales, por su parte, reconoció en diversas oportunidades que después de la muerte de Hugo Chávez y a raíz de la crisis venezolana, los proyectos conjuntos entre Yacimientos Petrolíferos Fiscales Bolivianos (YPFB) y Petróleos de Venezuela (PDVSA) se detuvieron, tomando el gobierno de Bolivia la batuta de las actividades (Morales, 2013). En el caso específico de Petroandina, la información sobre el desempeño de Venezuela en esta asociación y especialmente sobre los desembolsos de capital fue manejada por el gobierno boliviano bajo total hermetismo, existiendo además poca evidencia de una evolución positiva del acuerdo.

La escasa información oficial sobre los desembolsos o resultados de los acuerdos y/o proyectos de cooperación a partir de 2013 da indicios de una pérdida de interés por continuar con estas alianzas, especialmente en ámbitos estratégicos como el energético o productivo.

Por otra parte, no se puede dejar de lado al ALBA-TCP en un análisis de las relaciones de Venezuela con Ecuador y Bolivia en este periodo. Las relaciones de estos tres países dentro de este bloque tampoco mostraron mayores avances, más allá de los encuentros políticos para garantizar el apoyo a Venezuela en los organismos internacionales. Muchas de las empresas Gran-Nacionales que propuso el gobierno de Venezuela en Ecuador y Bolivia no lograron materializarse.

En 2013, con el ascenso de Nicolás Maduro a la presidencia de Venezuela, se trató de dar continuidad a varios acuerdos con Bolivia, entre ellos la Gran-Nacional textilera. De hecho, se llevó a cabo una reunión conjunta del entonces presidente del Banco del ALBA, Rafael Isea, y la entonces ministra de Desarrollo Productivo de Bolivia, Teresa Morales, en la que se acordó la creación de un fondo de operaciones de intercambio comercial de 29 millones de dólares para dar crédito a Enatex y al gremio textilero (República Bolivariana de Venezuela - Embajada en Bolivia, 2013).

Si bien fuentes oficiales enfatizaban en la importancia que tenía el mercado venezolano para los textiles bolivianos, a partir del 2014 comenzaron a generarse noticias sobre una realidad económica diferente para el gremio, especialmente dentro de la organización pública Enatex. La empresa finalmente pasó a llamarse Senatex en el 2016 después de una serie de problemas, que incluyó despidos y protestas. Con esta última situación se cerraba toda posibilidad de lograr la segunda y más ambiciosa fase del acuerdo, que era la creación de una Gran-Nacional de textiles bajo el marco del ALBA-TCP (Vergara, 2016, p. 85).

\footnotetext{
${ }^{20}$ El 14 de octubre de 2009 fue aprobada la "Donación” a Ecuador por parte de Venezuela de seis (06) aeronaves modelo Mirage-50 incluyendo partes y repuestos de éstas. Esta donación, según destaca el documento, se realizó bajo el marco del Convenio de Cooperación Técnico-Militar del ALBA.
} 
El caso de la Gran-Nacional Minera en Ecuador fue la única en materializarse, sin embargo, en 2016 el presidente Rafael Correa anunció la liquidación de la empresa (Correa, 2016), respondiendo a un escenario de contracción económica del país, pero también mostrando cierto pragmatismo político, necesario en una época de elecciones. La Gran-Nacional Minera, particularmente, representó una contradicción en el discurso del gobierno ecuatoriano, cuyo presidente manifestó en varias oportunidades su intención de pasar a un plano posextractivista (Ospina, 2013). Su cierre dejó una prueba más de la poca sostenibilidad de los proyectos que nacieron bajo el marco del ALBA-TCP.

Asimismo, después de la muerte de Hugo Chávez la información oficial del ALBA-TCP quedó prácticamente olvidada. El último boletín oficial del ALBA-TCP sobre avances de los proyectos y resultados estadísticos fue publicado en 2014. Los datos tampoco han sido alimentados con otros documentos oficiales de Ecuador y Bolivia.

Es importante resaltar, que el gobierno de Nicolás Maduro desde 2013, a diferencia de Hugo Chávez, no pudo mantener la misma dinámica en política exterior, en parte porque la crisis interna requirió mayor atención, pero también porque la época dorada para cooperar había terminado ante la merma de los petrodólares. Esto marcó un antes y un después en las relaciones con Ecuador y Bolivia. A lo anterior es imperativo agregar la falta de un ingrediente esencial, como fue el carisma del presidente Chávez (Romero y Mijares, 2016; Arenas; 2016), que terminó por difuminar el rol de Venezuela en la cooperación, especialmente para países como Ecuador y Bolivia.

\section{Conclusiones}

El rol de Venezuela en la CSS ha sido relevante, sobre todo por sus capacidades económicas vinculadas a la renta petrolera. Esta realidad no fue propia sólo del gobierno de Hugo Chávez, sino una práctica repetitiva de gobiernos precedentes. Sin embargo, en el siglo XXI las propuestas de cooperación de Venezuela con sus aliados políticos más cercanos en la región representaron un verdadero desafío al sistema de cooperación tradicional, independientemente de la sostenibilidad y más allá de logros concretos. El objetivo fundamental, que era medir fuerzas con la hegemonía estadounidense se cumplió, hasta cierto punto. El hecho de haber opacado a la propuesta estadounidense del Área de Libre Comercio de las Américas (ALCA) con alternativas como el ALBA-TCP, le dio un simbolismo histórico significativo, especialmente dentro de la lucha latinoamericana por conseguir un nuevo orden económico y político.

El ALBA-TCP (aunque no es el tema central de esta investigación) y las propuestas de cooperación venezolanas sirvieron en su momento para darle fuerza a un discurso político desafiante que encajó en las bases de la izquierda latinoamericana, cumpliendo además con el objetivo estratégico regional de su financiador para conseguir aliados.

A estas alturas es axiomático el uso que el gobierno de Venezuela hizo con su capacidad de financiamiento para asegurarse apoyo internacional, lo cual ha surtido efecto a largo plazo. Esto le resta crédito a su rol ejemplar dentro de la CSS. Sin embargo, como lo explica Zhenxing (2017, p. 11) aunque los gobiernos de la izquierda latinoamericana han cometido serios errores, no se puede negar que han dejado una importante "contribución histórica".

No obstante, estos hechos empezaron a perder relevancia ante un escenario político y económico tan complicado como el de la Venezuela de Nicolás Maduro. La crisis de Venezuela y las poco o nada acertadas políticas del gobierno actual (con una legitimidad cuestionada) han acrecentado las críticas a una alternativa progresista, convirtiendo al país caribeño en un mal ejemplo incluso para quienes una vez fueron sus aliados. Esto, ha repercutido negativamente en la imagen de la Venezuela cooperante.

El debate sobre el rol de Venezuela en la cooperación internacional quedó de lado, sobre todo porque su dinámica estaba vinculada con algunos elementos esenciales. Por un lado, el hiperpresidencialismo y el carisma de Hugo Chávez, quien se volcó a sostener una agenda 
internacional con generosos proyectos de cooperación regional con fines políticos y clientelares, sin mayores restricciones a nivel nacional. El enorme control que le fue conferido al presidente sobre las decisiones de interés nacional le permitió disponer de los recursos necesarios para promover sus propuestas, aunque al final éstas carecieran de control y seguimiento. Por otro lado, una bonanza petrolera que le permitió (por un tiempo) disponer de los fondos necesarios para impulsar su agenda de cooperación. Ante la ausencia de estos elementos, Venezuela pierde protagonismo en el escenario internacional.

En el entorno global, varios países (aparte de los ya tradicionales opositores al modelo de gobierno venezolano) se han pronunciado en contra de las medidas represivas que ha tomado Nicolás Maduro para mantenerse en el poder. Dentro de este contexto, la Bolivia de Evo Morales mantuvo una posición de apoyo hacia Venezuela incluso después de la muerte de Hugo Chávez, especialmente dentro de los organismos de concertación política, en parte, para poder mantener la base del discurso de su propio gobierno. Sin embargo, el gobierno de Morales también se mostró más pragmático, dejando las agendas con Venezuela, al menos los viejos proyectos de cooperación, en un plano olvidado.

Ecuador, por su parte, ya venía mostrando un paulatino distanciamiento desde la muerte de Hugo Chávez. Y aunque el ALBA-TCP (como plataforma de cooperación venezolana) no estuvo dentro de la agenda con mayor prioridad para el gobierno de Rafael Correa (prueba de ello es que nunca se suscribió al acuerdo del Banco del ALBA) es con el gobierno de Lenin Moreno que se cerró explícitamente el ciclo ALBA-TCP para el Ecuador y por supuesto cualquier posibilidad de CSS.

El gobierno de Lenin Moreno mantuvo por un tiempo su posición tibia en cuanto a los sucesos en Venezuela, tratando de proteger parte de su discurso progresista que, de alguna forma, le llevó al poder. Sin embargo, su posición es claramente más conservadora y por lo tanto resultaba conveniente marcar distancia con Venezuela, especialmente porque los intereses de Ecuador se dirigen hacia otro Norte. La política exterior del gobierno de Moreno ha dejado claro su interés por retomar las tradicionales relaciones políticas y comerciales con Estados Unidos y para ello era indispensable romper definitivamente con la administración chavistamadurista.

El (re)surgimiento de la derecha en la región debilitó aún más el apoyo hacia Venezuela, especialmente porque los cambios de gobiernos en los países aliados no resultaron favorables para el país caribeño, siendo un caso representativo el del Ecuador. Es así como los efectos de la cooperación venezolana, que hasta hace algunos años seguían teniendo eco en los países andinos para asegurarse apoyo político, han perdido fuerza.

Frente a estos cambios, es evidente que en la era post-Chávez solo quedan ruinas de un puñado de proyectos de cooperación fundamentados en petrodólares y por lo tanto insostenibles para Venezuela, Ecuador y Bolivia. La falta de seguimiento institucional, de transparencia y de vigilancia ciudadana, incluso durante el gobierno de Chávez, contribuyeron a dirigir la cooperación a este final. Ciertamente, hubo casos exitosos sobre todo en materia social que no se pueden obviar, como las Misiones a través del ALBA-TCP, y que pudieran presentarse como un ícono para la CSS venezolana. Pero corrieron, a la larga, la misma suerte que los proyectos estratégicos en el ámbito energético, financiero y comercial.

\section{Referencias}

Acosta, A. (2010). El Buen Vivir en el camino del post-desarrollo. Una lectura desde la Constitución de Montecristi, en Policy Paper, Fundación Friedrich Ebert. Quito: FES-ILDIS

ALBA-TCP (2014). Informe de Gestión de la Secretaría del ALBA 2004-2014. Recuperado de http://albatcp.org/public/documents/decimo/Espanol/INFORME de gestion.pdf. Consulta: 16 de enero de 2015 
Aponte García, M. (2014). El nuevo regionalismo estratégico. Los primeros diez años del ALBATCP. Buenos Aires: CLACSO

Aponte, M. (2015). La teorización del nuevo regionalismo estratégico en el ALBA-TCP, en Aponte, M. y Amézquita Puntiel, G. (comp). El ALBA-TCP origen y fruto del nuevo regionalismo latinoamericano y caribeño. Buenos Aires, Argentina: CLACSO

Arenas, N. (2016). Nicolás Maduro: ¿populismo sin carisma?, en Cuadernos del CENDES, 33 (92), pp. 113-128, Caracas, Venezuela

Asociación Civil Control Ciudadano (2016). Acuerdos de Cooperación Militar 2005-2016. Estudio de caso III. Venezuela. Recuperado de http://controlciudadano.org/web/wpcontent/uploads/VENEZUELA_ACUERDOS-DE-COOPERACION-II-Estudio.pdf.

Consulta: 13 de marzo 2016

Ayllón Pino, B. (2012). Cooperación Internacional para el Desarrollo (CID) SUR-SUR: América Latina y sus otros vecinos, en Las Relaciones Internacionales de América Latina: desafíos estratégicos y oportunidades de aprendizaje. Instituto Universitario de Desarrollo y Cooperación, Salamanca, España

Ayllón Pino, B. (2015). La Cooperación Sur-Sur en América Latina y el Caribe. De una época dorada a una fase incierta. En Serbin, A., Martínez, L. y Ramanzini J., H. América Latina y el Caribe y el nuevo sistema internacional: Miradas desde el Sur. Anuario de la Integración Regional de América Latina y el Caribe, 11, pp. 134-170. Buenos Aires, Argentina: CRIES

Benzi, D. (2016). El exitoso ocaso del ALBA, Nueva Sociedad, 261, pp. 77-91

Benzi, D., Guayasamín, T. y Vergara, M. (2016). ¿Hacia una Nueva Arquitectura Financiera Regional? Problemas y perspectivas de la cooperación monetaria en el ALBA-TCP. Iberoamerican Journal of Development Studies, 1 (5), pp. 32-61

Briceño Ruiz, J. (2013). Ejes y modelos en la etapa actual de la integración económica regional en América Latina. Estudios Internacionales, 175, pp. 9-39

Briceño Ruiz, J. (2015). ¿Gobernanza regional o soft-balancing de "Estados revolucionarios"?: El discurso y la práctica del ALBA. Pensamiento Propio, 42, pp. 167-194

Caballero, S. (2012). La cooperación Sur-Sur en relación con los proyectos de integración sudamericanos. En Carou, H., Cabezas, A., Mallo, T., Del Campo, E. y Carpio, J. (edit). XV Encuentro de Latinoamericanistas Españoles, pp. 1247-1252. Madrid, España: Trama Editorial CEEIB

Chomsky, N. (2002). Estados canallas. El imperio de la fuerza en los asuntos mundiales. Buenos Aires, Argentina: Paidós

Cobo, L. (2008). Venezuela y el mundo transnacional: Instrumentación de la política exterior venezolana para imponer un modelo en América Latina. Caracas, Venezuela: ILDIS

Comisión Económica para América Latina y el Caribe (2010). La cooperación internacional en el nuevo contexto mundial: reflexiones desde América Latina y el Caribe. Disponible en https://repositorio.cepal.org/bitstream/handle/11362/13434/S2010972.pdf?sequence=1\&isAl lowed=y. Consulta: 18 de febrero de 2020.

Contraloría General del Estado (2014). Auditoría externa DP Manabí DRSDPM-AE-0051-2014. Recuperado de http://www.contraloria.gob.ec/buscar.asp. Consulta: 23 de mayo de 2015

Corrales, J. \& Penflod, M. (2011). Dragon in the tropics. Hugo Chávez and the Political Economy of Revolution in Venezuela. Whashington DC, USA: Brooking Institution Press

Corrales, J. (2009). Using Social Power to Balance Soft Power: Venezuela's Foreign Policy. The Washington Quarterly, 32 (4), pp. 97-114

Correa, R. (2016). Informe a la Nación. TeleAmazonas, 24 de mayo de 2016. Recuperado de http://www.teleamazonas.com/2016/05/presidente-correa-presento-ultimoinforme-lanacion-la-asambleal. Consulta: 26 de mayo de 2016 
Cusack, A. K. (2015). ALBA, el Tratado de Comercio de los Pueblos y los obstáculos persistentes a la cooperación económica Sur-Sur en América Latina y el Gran Caribe. En Serbin, A., Martínez, L. y Ramanzini J., H. América Latina y el Caribe y el nuevo sistema internacional: Miradas desde el Sur. Anuario de la Integración Regional de América Latina y el Caribe, 11, pp. 187-212. Buenos Aires, Argentina: CRIES

De Grave, J. (2007). Estado boliviano y Cooperación Internacional. Malestar, responsabilidades, aperturas. En Documento de trabajo. Informe Nacional sobre el Desarrollo en Bolivia, PNUD

Decreto Supremo 196 (2009). Gaceta Oficial, Estado Plurinacional de Bolivia, 8 de julio. Recuperado de http://www.gacetaoficialdebolivia.gob.bo. Consulta: 18 de febrero de 2019

Domínguez, R. (2015). La cooperación económica y Financiera de Venezuela como superpotencia de Cooperación Sur-Sur. Comentario Internacional, 15, pp. 89-117

Falconí, F. (2010). ¡Con Ecuador por el Mundo! La política internacional ecuatoriana. Quito, Ecuador: Editorial el Conejo

Goldwyn, D. L. \& Gill, C. R. (2014). Uncertain energy: The Caribbean's gamble with Venezuela. Washington, USA: The Atlantic Council's Adrienne Arsht Latin America Center

Gran-Nacional Minera Mariscal Sucre C.E.M (2014). Kit de Prensa. Recuparado de http://www.grannacionalminera.com/noticias/descargas/category/2-informativos.html. Consulta: 22 de julio de 2015

Guzmán, P. (2011). Entrevista con Freddy Morales. TeleSUR, 31 de marzo de 2011, La Paz. Recuperado de https://www.youtube.com/watch?v=83J9NvBjiFg. Consulta: 31 de marzo de 2016

Instituto Nacional de Estadística de Bolivia (2018). Encuesta de Hogares: Pobreza en Bolivia baja durante el 2017. Recuperado de https://www.ine.gob.bo/index.php/notas-de-prensa-ymonitoreo/itemlist/tag/Pobreza. Consulta: 23 de junio de 2019

Instituto Nacional de Estadística de Ecuador (2016). Reporte de Pobreza y Desigualdad. Recuperado de https://www.ecuadorencifras.gob.ec/documentos/webinec/POBREZA/2016/Diciembre_2016/Reporte\%20pobreza\%20y\%20desigualdaddic16.pdf. Consulta: 23 de junio de 2019

Ley 167 (2011). Acuerdo de Comercio entre los Pueblos y Complementariedad Económica y Productiva, entre el Gobierno de la República Bolivariana de Venezuela y el Estado Plurinacional de Bolivia. Estado Plurinacional de Bolivia, 19 de agosto de 2011. Recuperado de https://www.aduana.gob.bo. Consulta: 31 de marzo de 2015

Llanos, M. \& Nolte, D. (2016). The many faces of Latin American presidentialism. GIGA Focus Lateinamerika, 1, German Institute of Global and Area Studies- Leibniz-Institut für Globale und Regionale Studien, Hamburg

López C., S. (2014). Cronología e historia de la cooperación sur-sur. Un aporte desde Iberoamérica. Documento de trabajo, $\mathrm{N}^{\circ}$ 5, Programa Iberoamericano para el fortalecimiento de la Cooperación Sur-Sur

Mawdsley, E. (2019). South-South Cooperation 3.0? Managing the consequences of success in the decade ahead. Oxford Development Studies, 47 (3), pp. 259-274

Ministerio de Relaciones Exteriores y Movilidad Humana de la República del Ecuador (2009). Sistema de Tratados. Recuperado de http://sitrac.cancilleria.gob.ec/sitrac/consultas/busqueda.aspx. Consulta: 23 de abril de 2016

Ministerio de Relaciones Exteriores y Movilidad Humana de la República del Ecuador (2014). Sistema de Tratados. Recuperado de http://sitrac.cancilleria.gob.ec/sitrac/consultas/busqueda.aspx. Consulta: 23 de abril de 2016

Morales Ayma, E. (2013). Entrevista con Claudia Palacios en "Los Influyentes". CNN en español, 12 de abril de 2013. Recuperado de https://www.youtube.com/watch?v=k3Da -gCSjk. Consulta: 31 de marzo de 2016 
Muhr, T. (2011). Venezuela and the ALBA: Counter-Hegemony, Geographies of Integration and Development, and Higher Education for all. VDM/Akademikerverlag, Saarbrücken

Muhr, T. (2012) The Politics of Space in the Bolivarian Alliance for the Peoples of Our AmericaPeoples' Trade Agreement (ALBA-TCP): Transnationalism, the Organized Society, and Counter-Hegemonic Governance. Globalizations, 9(6), pp. 767- 782

Muhr, T. (2015). La construcción transnacional del ALBA-TCP en El Salvador, Nicaragua y los Estados Unidos de América. Contra-globalización y una política revolucionaria de lugar, espacio y escala. En Aponte, M. y Amézquita, G. (comp). El ALBA-TCP origen y fruto del nuevo regionalismo latinoamericano y caribeño, pp. 25-68. Buenos Aires, Argentina: CLACSO

Ojeda, T. (2010). Experiencias Venezolanas en Cooperación Sur-Sur. En Ayllón, B. \& Surasky, J. (coords). La cooperación Sur-Sur en Latinoamerica, utopía y realidad. Madrid, España: Editorial Catarata / IUDC-UCM

Organización de las Naciones Unidas (2009). Documento final de Nairobi de la Conferencia de Alto Nivel de las Naciones Unidas sobre la Cooperación Sur Sur. Resolución aprobada por la Asamblea General el 21 de diciembre de 2009. Recupareado de https://undocs.org/pdf?symbol=es/A/RES/64/222. Consulta: 21 de mayo de 2015

Ospina, P. (2013). Estamos haciendo mejor las cosas con el mismo modelo antes que cambiarlo. La Revolución Ciudadana en Ecuador (2007-2012). En Lander, E., Arze, C., Gómez, J., Ospina, P. y Álvarez, V. (comp). Promesas en su laberinto: Cambios y continuidades en los gobiernos progresistas de América Latina, pp. 177-277. La Paz, Bolivia: IEE/CEDLA/CIM

Pape, R. A. (2005). Soft Balancing against the United States, International Security, 30 (1), pp. 7-45

Paredes, J. (2013). Petroandina dice que invertirá lo comprometido. La Razón, 10 de abril, La Paz. Recuperado de http://www.la-razon.com/economia/Petroandina-dice-invertiracomprometido_0_1812418776.html. Consulta: 09 de marzo de 2015

Paz A., V. (2016). El Financiamiento del Desarrollo en Bolivia. Cambios y Continuidades en la Relación Estado Cooperación Internacional (2006-2013). Documento de trabajo 2016-15, Instituto de Investigación de las Naciones Unidas para el Desarrollo Social (UNRISD). Recuperado http://www.unrisd.org/80256B3C005BCCF9/(httpAuxPages)/0902E98FFB568EBFC12580 90005AF0C7/\$file/Paz.pdf. Consulta: 31 de marzo de 2016

Penflod, M. (2010). La democracia subyugada: El hiperpresidencialismo venezolano. Revista de ciencia Política 30, (1), pp. 21-40

Petersen, C. (2012). Programas del ALBA-TCP en Bolivia. Independent Study Project (ISP) Collection, Paper 1303

Petroandina S.A.M. (2014). Memoria anual 2013-2014. Recuperado de http://ypfbpetroandina.com.bo/wp-content/uploads/2015/02/MEMORIA-YPFBPETROANDINA-colores.compressed.pdf. Consulta: 12 de abril de 2016

Ponce, J. y Onofa, M. (2009). La Alfabetización en el Ecuador. Evolución histórica, información actualizada y mapa nacional del analfabetismo.Quito, Ecuador: UNESCO

República Bolivariana de Venezuela - Embajada en Bolivia. (2013). Banco del Alba aumenta crédito a textilera boliviana Enatex. Recuperado de http://bolivia.embajada.gob.ve/?option=com_content\&view=article\&id=1937\%3Abancodel-alba-aumenta-credito-a-textilera-boliviana-enatex\&catid=3\%3Anoticias-de-venezuelaen-el-mundo\&Itemid=19\&lang=es. Consulta: 31 de marzo de 2016

Ribando S., C. (2008). Bolivia: Political and Economic Developments and Relations with the United States. Washington, USA: Congressional Research Service

Riggirozzi, P. (2012). Re-territorializando consensos: Hacia un regionalismo post-hegemónico en América Latina. En Serbin, A., Martínez, L., \& Ramanzini J. H. (coord.). El regionalismo 
"post-liberal" en América Latina y el Caribe: Nuevos actores, nuevos temas, nuevos desafíos, pp. 129-152. Buenos Aires: CRIES

Romero, C. y Curiel, C. (2009). Venezuela: Política exterior y rentismo. Cadernos PROLAM/USP, año 8 (9), pp. 39-61

Romero, C., \& Mijares, J. (2016). From Chávez to Maduro: Continuity and Change in Venezuelan Foreign Policy. Contexto Internacional, 38 (1), pp. 165-201

Roncal V., X. (2013). ALBA-TCP como estrategia de integración alternativa para el desarrollo de América Latina y el Caribe. La Paz, Bolivia: Instituto Internacional de Integración Convenio Andrés Bello

Sanahuja, J. A. (2009). Del 'regionalismo abierto' al 'regionalismo post-liberal'. Crisis y cambio en la integración regional en América Latina y el Caribe. En Martínez, L., Peña, L. y Vázquez, M. (coord). Barreras y obstáculos a la integración en América Latina y el Caribe, pp. 1154. Buenos Aires, Argentina: CRIES

Secretaría Ejecutiva del Consejo Monetario Regional del SUCRE (2013). Informe de Gestión 2013. Recuperado de http://www.sucrealba.org/images/informes/informe_2012.pdf. Consulta: 13 de septiembre de 2015

Secretaría General Iberoamericana (2008). II Informe de la Cooperación Sur-Sur en Iberoamerica, SEGIB, PentaCrom

Serbín, A. y Serbín Pont, A. (2014). Quince años de política exterior bolivariana: ¿entre el softbalancing y la militarización? Pensamiento Propio, año 19 (39), pp.287-326

The Reality of Aid (2010). Cooperación Sur-Sur: Un desafío al sistema de la Ayuda. Medellín, Colombia: Fundación Ibon

Toro, A. (2011). El ALBA como instrumento de "soft-balancing". Pensamiento Propio, año 16 (33), pp.159-183

UNESCO (2013). Situación Educativa de América Latina y el Caribe: Hacia la educación de calidad para todos al 2015, Oficina Regional de Educación para América Latina y el Caribe (OREALC/UNESCO), Santiago. Recuperado de http://www.unesco.org/new/fileadmin/MULTIMEDIA/FIELD/Santiago/images/SITIEDespanol.pdf. Consulta: 13 de abril de 2016

Vallejo, R. (2009). Ecuador no es territorio libre de analfabetismo. El Comercio, 21 de octubre de 2009, Recuperado de http://www.elcomercio.com/actualidad/ecuador-no-territoriolibreanalfabetismo.html. Consulta: 04 de abril de 2015

Vergara, M. (2016). Evolución de la cooperación venezolana con Ecuador y Bolivia en el periodo 2006-2014 (Tesis de Maestría). Universidad Andina Simón Bolívar, Quito, Ecuador

Viceministerio de Inversión Pública y Financiamiento Externo (VIPFE) (2013). La Cooperación Internacional en Bolivia 2013. La Paz, Bolivia: Ministerio de Planificación del Desarrollo

Villaroel, G. E. y Ledezma, N. (2007). Carisma y política. El liderazgo de Hugo Chávez desde la perspectiva de sus partidarios. Politeia, 30 (39), pp. 1-22. Universidad Central de Venezuela, Caracas

Villavicencio, F. (2013). Ecuador: Made in China. Quito, Ecuador: InterAmerican Institute for Democracy

Zhenxing, S. (2017). China and Latinamaerica. Economic and trade Cooperation in the next ten years. China Academy of Social Science. London, England: World Scientific Publishing 